\title{
Distributed estimation based on quantized data
}

\author{
Yoon Hak Kim ${ }^{\text {a) }}$ \\ System LSI Division, Samsung Electronics, Giheung campus, Gyeonggi-Do, Korea \\ $446-711$ \\ a)yhk418@gmail.com
}

Abstract: Since standard statistical estimation methods are built on the models that treat numerical data as continuous variables, they can be inappropriate and misleading when quantization process is involved in estimation. In this paper, we propose novel distributed estimation algorithms based on the Maximum Likelihood (ML) method. Motivated by the observation that each quantized measurement corresponds to a region with which the parameter to be estimated is associated, we develop algorithms that estimates the likelihood of each of the regions rather than that of the parameter itself. Our simulation results show that the proposed algorithms achieve good performance as compared with traditional ML estimators.

Keywords: distributed estimation, Maximum Likelihood (ML), Maximum A Posteriori (MAP), Kullback-Leibler (KL) divergence

Classification: Science and engineering for electronics

\section{References}

[1] R. Niu and P. K. Varshney, "Target Location Estimation in Wireless Sensor Networks using Binary Data," Proc. 38th Annual Conference on Information Sciences and Systems, March 2004.

[2] J.-J. Xiao, A. Ribeiro, Z.-Q. Luo, and G. B. Giannakis, "Distributed Compression-Estimation Using Wireless Sensor Networks," IEEE Signal Process. Mag., vol. 23, no. 4, pp. 27-41, July 2006.

[3] S. B. Vardeman and C.-S. Lee, "Likelihood based statistical estimation from quantized data," IEEE Trans. Instrum. Meas., vol. 54, no. 1, pp. 409-414, Feb. 2005.

[4] P. H. Garthwaite, I. T. Jolliffe, and B. Jones, "Statistical Inference Second Edition," Oxford University Press, 2002.

[5] D. Li and Y. H. Hu, "Energy-Based Collaborative Source Localization Using Acoustic Microsensor Array," EURASIP Journal on Applied Signal Processing, pp. 321-337, 2003.

[6] Y. H. Kim and A. Ortega, "Maximun A Posteriori (MAP)-based Algorithm for Distributed Source Localization using Quantized Acoustic Sensor Readings," IEEE ICASSP, May 2006.

[7] Y. H. Kim and A. Ortega, "Quantizer Design for Source Localization in Sensor Networks," IEEE ICASSP, March 2005. 


\section{Introduction}

In literature $[1,2,6]$, estimation problem has been addressed for distributed systems where $M$ distributed nodes sense measurements on the unknown parameter to be estimated, $\theta \in S \subset \mathbf{R}^{N}$ :

$$
x_{i}=f_{i}(\theta)+\omega_{i}, \quad i=1, \ldots, M
$$

where $x_{i}$ is the measurement at node $i, f_{i}(\theta)$ is the sensing model employed at node $i$ and $\omega_{i}$ is the measurement noise approximated by normal distribution $N\left(0, \sigma_{i}^{2}\right)$. It is assumed that each $x_{i}$ is conditionally independent given $\theta$; formally,

$$
p\left(x_{1}, \ldots, x_{M} \mid \theta\right)=\prod_{i=1}^{M} p\left(x_{i} \mid \theta\right) \quad \text { where } \quad p\left(x_{i} \mid \theta\right)=N\left(f_{i}(\theta), \sigma_{i}^{2}\right) .
$$

The classical Maximum Likelihood Estimator (MLE) is given by [4]

$$
\hat{\theta}_{M L}=\arg \max _{\theta} L\left(\theta ; x_{1}^{M}\right), \quad x_{1}^{M}=\left(x_{1}, \ldots, x_{M}\right)
$$

where the likelihood function $L\left(\theta ; x_{1}^{M}\right)$ is given by $p\left(x_{1}^{M} \mid \theta\right)$. In practice, often the log-likelihood function is maximized rather than the likelihood function, primarily for mathematical convenience because the monotonicity of the logarithmic function yields the same estimated values. The log-likelihood function is written by

$$
l\left(\theta ; x_{1}^{M}\right)=\ln L\left(\theta ; x_{1}^{M}\right)=\sum_{i} \ln p\left(x_{i} \mid \theta\right)=\sum_{i}\left[\ln \left(\frac{1}{\sqrt{2 \pi \sigma_{i}}}\right)+\left(-\frac{\left(x_{i}-f_{i}(\theta)\right)^{2}}{2 \sigma_{i}^{2}}\right)\right] .
$$

While the classical MLE had been developed based on the frequency-based theory which treats parameters as fixed but unknown, it can be easily combined with the information contained in $p(\theta)$ using Bayes' theorem. That is, if the prior distribution $p(\theta)$ is available, it would be incorporated into the estimation process as follows: denoting such an estimator the Bayesian MLE, we have

$$
\begin{aligned}
\hat{\theta}_{B M L} & =\arg \max _{\theta} L\left(\theta ; x_{1}^{M}\right) p(\theta)=\arg \max _{\theta} p\left(x_{1}^{M} \mid \theta\right) p(\theta) \\
& =\arg \max _{\theta} \prod_{i=1}^{M}\left(\frac{1}{\sqrt{2 \pi \sigma_{i}}}\right) \exp \left(-\frac{\left(x_{i}-f_{i}(\theta)\right)^{2}}{2 \sigma_{i}^{2}}\right) p(\theta) .
\end{aligned}
$$

Notice that the Bayesian MLE maximizes the posterior distribution $p\left(\theta \mid x_{1}^{M}\right)=p\left(x_{1}^{M} \mid \theta\right) p(\theta) / p\left(x_{1}^{M}\right)$ given the measurements $x_{1}^{M}$. Clearly, when there is full ignorance about $\theta$ which is frequently used in practice, the classical MLE is equivalent to the Bayesian MLE.

It is noted that quantization of measurements is typically ignored as methods of statistical inference are developed while quantization process becomes an indispensable part of practical systems, especially power-constrained systems such as sensor networks where each quantized measurement is transmitted to a fusion center which produces $\hat{\theta}$, an estimate of the parameter from 
quantized measurements. In other words, most standard statistical methods are built on the models that interpret numerical data as continuous variables, which can render those methods inappropriate and misleading [3]. In this work, we focus on how the likelihood function can be appropriately expressed when quantized data are involved in estimation and propose novel distributed estimators along with their optimality criteria which can be efficiently used for obtaining good estimators with reduced complexity as compared with the minimum mean squared error (MMSE) estimator which is optimal in a sense of minimizing the estimation error $E\|\theta-\hat{\theta}\|^{2}$ but is avoided for practical systems because of its high computational complexity [6].

\section{Likelihood functions from quantized data}

Denote $Q_{i}$ a quantized measurement at node $i$ representing an interval $\left[\begin{array}{ll}Q_{i}^{l} & Q_{i}^{h}\end{array}\right]$ that measurement $x_{i}$ belongs to. Then we express the likelihood function, $L_{Q}$ in terms of quantized data as follows:

$$
\begin{aligned}
L_{Q}\left(\theta ; Q_{1}^{M}\right) & =p\left(Q_{1}^{M} \mid \theta\right)=\prod_{i} p\left(Q_{i} \mid \theta\right)=\prod_{i} \int_{\theta \in Q_{i}} p\left(x_{i} \mid \theta\right) d x_{i} \\
& =\prod_{i}\left[\Phi\left(\frac{Q_{i}^{h}-f_{i}(\theta)}{\sigma_{i}}\right)-\Phi\left(\frac{Q_{i}^{l}-f_{i}(\theta)}{\sigma_{i}}\right)\right]
\end{aligned}
$$

where $Q_{1}^{M}=\left(Q_{1}, \ldots, Q_{M}\right)$ and $\Phi(\cdot)$ is the cumulative distribution function (cdf) for the normal distribution, $N(0,1)$. Similarly, the corresponding loglikelihood function is given by

$$
\begin{aligned}
l_{Q}\left(\theta ; Q_{1}^{M}\right) & =\ln p\left(Q_{1}^{M} \mid \theta\right)=\sum_{i} \ln p\left(Q_{i} \mid \theta\right) \\
& =\sum_{i} \ln \left[\Phi\left(\frac{Q_{i}^{h}-f_{i}(\theta)}{\sigma_{i}}\right)-\Phi\left(\frac{Q_{i}^{l}-f_{i}(\theta)}{\sigma_{i}}\right)\right] .
\end{aligned}
$$

\section{Proposed algorithms}

It is observed that when quantization of measurements are involved in estimation process, a region of the parameters to be estimated is very likely to induce the same quantized measurements, especially in the high SNR regime. Assuming $\omega_{i}=0$ in (1), we can deterministically construct a region $A$ associated with any given combination of $M$-tuple, $Q_{1}^{M}=\left(Q_{1}, \ldots, Q_{M}\right)$ :

$$
A=\left\{\theta \mid f_{i}(\theta) \in Q_{i}=\left[Q_{i}^{l} \quad Q_{i}^{h}\right], i=1, \ldots, M\right\} .
$$

This will motivate us to first find the region of the parameter that is the most likely to occur rather than the parameter itself. The extent of likeliness of such regions can be measured by the average likelihood function, which is defined as follows: denoting $L_{a}\left(A_{j} ; Q_{1}^{M}\right)$ (or $\left.l_{a}\left(A_{j} ; Q_{1}^{M}\right)\right)$ the average likelihood (or average log-likelihood) for the $j$-th region $A_{j}$,

$$
L_{a}\left(A_{j} ; Q_{1}^{M}\right) \equiv E_{A_{j}}\left[L_{Q}\left(\theta ; Q_{1}^{M}\right) p_{j}\right]=\int_{\theta \in A_{j}} p\left(Q_{1}^{M} \mid \theta\right) p\left(\theta \mid \theta \in A_{j}\right) p_{j} d \theta
$$




$$
l_{a}\left(A_{j} ; Q_{1}^{M}\right) \equiv E_{A_{j}} \ln \left[L_{Q}\left(\theta ; Q_{1}^{M}\right) p_{j}\right]=\int_{\theta \in A_{j}} \ln \left[p\left(Q_{1}^{M} \mid \theta\right) p_{j}\right] p\left(\theta \mid \theta \in A_{j}\right) d \theta
$$

where $p_{j}=p\left(\theta \in A_{j}\right)$. Clearly, the average likelihood function $L_{a}\left(A_{j} ; Q_{1}^{M}\right)$ is a measure of average likeliness for the parameter $\theta \in A_{j}$ to actually induce $Q_{1}^{M}$. Later in the experiments in Section 5, we show that the average likelihood function can capture information on the parameter more efficiently than the standard likelihood function. Then, we propose new estimators that exploits these average likelihood functions as follows:

$$
\begin{aligned}
& A_{L}^{*}=\arg \max _{j} L_{a}\left(A_{j} ; Q_{1}^{M}\right) \quad \hat{\theta}_{M A L Q}=E\left(\theta \mid \theta \in A_{L}^{*}\right) \\
& A_{l}^{*}=\arg \max _{j} l_{a}\left(A_{j} ; Q_{1}^{M}\right) \quad \hat{\theta}_{M A L L Q}=E\left(\theta \mid \theta \in A_{l}^{*}\right)
\end{aligned}
$$

where the subscript MALQ (MALLQ) represents Maximum Average Likelihood (Log-Likelihood) from Quantized data. While the likelihood function and the corresponding log-likelihood function lead to the same estimator in classical ML estimation, the estimators obtained from our proposed formulations where the likelihood functions are averaged over regions, may produce different results since they are optimized using two different metrics. In the perspective of optimality, our proposed estimators will be shown to achieve their own optimality, respectively.

\section{Performance evaluation: optimality criteria}

\subsection{Hypothesis testing problem}

Once the parameter space $S$ is divided into disjoint regions, each of which can be constructed from (5), the estimation problem will be formulated as the hypothesis testing problem by denoting $H_{j}$ the $j$-th hypothesis corresponding to the $j$-th region $A_{j}$. Our goal is then to find $H^{*}$ that maximizes the posterior distribution given the received noisy M-tuple $Q_{1}^{M}$ as follows:

$$
\begin{aligned}
H^{*} & =\arg \max _{j} p\left(H_{j} \mid Q_{1}^{M}\right)=\arg \max _{j} p\left(Q_{1}^{M} \mid \theta \in A_{j}\right) p_{j} \\
& =\arg \max _{j} \int_{\theta \in A_{j}} p\left(Q_{1}^{M} \mid \theta\right) p\left(\theta \mid \theta \in A_{j}\right) p_{j} d \theta \\
& =\arg \max _{j} E_{A_{j}} L_{Q}\left(\theta ; Q_{1}^{M}\right) p_{j}=\arg \max _{j} L_{a}\left(A_{j} ; Q_{1}^{M}\right) .
\end{aligned}
$$

Once $H^{*}$ is obtained, the estimate $\hat{\theta}$ is computed by $E\left(\theta \mid H^{*}\right)=E(\theta \mid \theta \in$ $A_{L}^{*}$ ). Clearly, $\hat{\theta}$ that is optimal in Maximum A Posteriori (MAP) sense [6], is equivalent to $\hat{\theta}_{M A L Q}$ given by (8).

\subsection{Kullback-Leibler (KL) divergence}

Since estimation is regarded as a process of combating noise buried in the measurements and finding the actual measurements which would have been obtained without noise, we are tempted to find those actual quantized measurements from the noisy quantized ones. To accomplish this task, we first introduce each posterior distribution corresponding to one of the noise-free measurements, $Q_{1}^{M}(j), \forall j$ :

$$
p\left(\theta \mid Q_{1}^{M}(j)\right)=p\left(\theta \mid \theta \in A_{j}\right), \quad \forall j
$$


where $A_{j}$ is constructed from $Q_{1}^{M}(j)$ by using (5). That is, $Q_{1}^{M}(j)$ corresponds to the $M$ quantized measurements generated from all $\theta \in A_{j}$ with $\omega_{i}=0$ in (1). Note that $p\left(Q_{1}^{M}(j) \mid \theta\right)=p\left(\theta \in A_{j} \mid \theta\right)=0$ for all $\theta \in S-A_{j}$. Such posterior distributions are assumed to be contaminated with the measurement noise and to be transmitted to the fusion center. Then, we seek to acquire the actual noise-free measurement by finding the posterior distribution that is the most similar to that of the received noisy M-tuple, $Q_{1}^{M}$. With this in mind, we define the distortion measure between $Q_{1}^{M}(j)$ and $Q_{1}^{M}$ using Kullback-Leibler (KL) divergence as follows:

$$
\begin{aligned}
d\left(Q_{1}^{M}(j), Q_{1}^{M}\right) & =D_{K L}\left[p\left(\theta \mid Q_{1}^{M}(j) \| p\left(\theta \mid Q_{1}^{M}\right)\right]\right. \\
& =\int_{\theta \in S} p\left(\theta \mid Q_{1}^{M}(j)\right) \ln \frac{p\left(\theta \mid Q_{1}^{M}(j)\right)}{p\left(\theta \mid Q_{1}^{M}\right)} d \theta \\
& =\int_{\theta \in S} p\left(\theta \mid \theta \in A_{j}\right) \ln \left[\frac{p\left(Q_{1}^{M}(j) \mid \theta\right) p\left(Q_{1}^{M}\right)}{p\left(Q_{1}^{M} \mid \theta\right) p\left(Q_{1}^{M}(j)\right)}\right] d \theta \\
& \propto-\int_{\theta \in A_{j}} p\left(\theta \mid \theta \in A_{j}\right) \ln \left[p\left(Q_{1}^{M} \mid \theta\right) p_{j}\right] d \theta \\
& =-l_{a}\left(A_{j} ; Q_{1}^{M}\right)
\end{aligned}
$$

where (12) follows from (11). Note that $D_{K L}(p \| q)$ is a measure of the distance between two distributions, $p, q$.

Next, we search for the noise-free measurement that minimizes the distortion measure and obtain the estimator by taking the mean of the corresponding posterior distribution. Formally,

$$
\begin{aligned}
Q_{1}^{M}(*) & =\arg \min _{j} d\left(Q_{1}^{M}(j), Q_{1}^{M}\right)=\arg \max l_{a}\left(A_{j} ; Q_{1}^{M}\right) \\
\hat{\theta} & =E\left[\theta \mid Q_{1}^{M}(*)\right]=E\left[\theta \mid \theta \in A_{l}^{*}\right] .
\end{aligned}
$$

Obviously, the distortion measure indicates the negative of the average loglikelihood function, implying that $\hat{\theta}$, optimal in a sense of minimizing the KL divergence, is equivalent to $\hat{\theta}_{M A L L Q}$. As shown in (10) and (12), the computational complexity to obtain $\hat{\theta}_{M A L Q}$ or $\hat{\theta}_{M A L L Q}$ is quite acceptable for practical systems as compared with the MMSE estimator which requires integration over $S[6]$. It is noted that under noise-free conditions $\left(\omega_{i}=0\right)$, our two estimators would be equivalent to the MMSE estimator since the actual measurements are recovered without error from the received M-tuple. In addition, as the rate approaches infinity, they would be asymptotically reduced to the ML estimator.

\section{Simulation results}

In our experiments, we consider source localization in a sensor network where $M=5$ sensors are deployed randomly in a $10 \times 10 \mathrm{~m}$ sensor field. Each sensor measures an acoustic source energy based on the energy decay model [5], quantizes it using a quantizer with rate $R_{i}$ designed by the algorithm in [7] and sends the quantized measurement to a fusion center which performs localization using distributed estimation algorithms. The measurement noise 
is assumed to be normal distributed, $N\left(0, \sigma^{2}\right)$ and SNR is computed by $10 \log _{10} \frac{a^{2}}{\sigma^{2}}$ where $a$ is the source signal energy. Note that SNR is measured at 1 meter from the source. For practical vehicle target, the SNR is often much higher than $\mathrm{SNR}=40 \mathrm{~dB}$ which corresponds to $\mathrm{SNR} \approx 5.5 \mathrm{~dB}$ measured at each sensor on the average [5].

The proposed algorithms described in Section 3 are evaluated in terms of localization error $E\|\theta-\hat{\theta}\|^{2}$ for 100 different sensor configurations. For each configuration, a test set of 2000 source locations are generated with a uniform distribution of $p(\theta)$ to compute $E\|\theta-\hat{\theta}\|^{2}$ by varying SNR and rate $R_{i}$. In Figure 1 the proposed algorithms are compared with typical standard ML estimation and MMSE estimation which serves as a good lower bound. It should be pointed out that our algorithms provide good localization accuracy as compared with the typical ML estimator and achieves the performance close to that of the MMSE estimator, especially at high SNR.
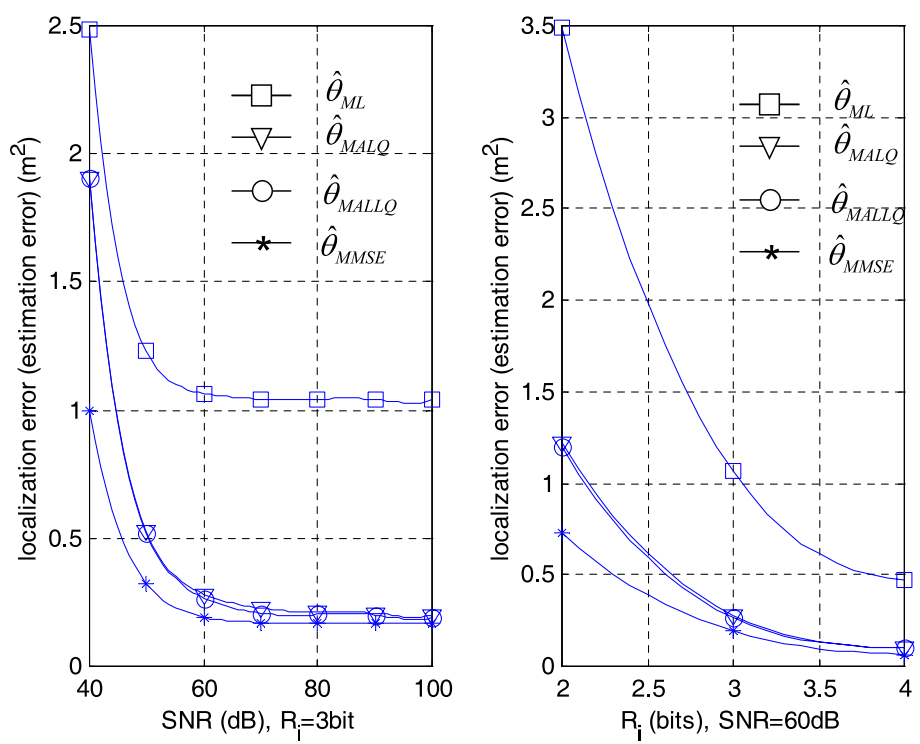

Fig. 1. Comparison of proposed algorithms with MMSE and ML estimations. SNR $=40, \ldots, 100 \mathrm{~dB}$ with $R_{i}=3$ (left) and $R_{i}=2,3$ and 4 bits with $\mathrm{SNR}=60 \mathrm{~dB}$ (right).

\section{Conclusion}

In this paper, we have proposed novel distributed estimation algorithms that exploit the average likelihood function as a metric since it captures information contained in quantized data more efficiently than the likelihood function. We also provided optimality criteria for the proposed algorithms, each of which was shown to achieve its optimality. Experimental results demonstrated that our estimators performed well with respect to the classical ML estimator and achieved the performance close to that of the MMSE estimator without incurring high complexity. 